\title{
Defective pneumococcal antibody response in patients with recurrent respiratory tract infections
}

\author{
Baran Erman ${ }^{1,2}$, Duygu Demirtaş ${ }^{3}$, Hacer Neslihan Bildik ${ }^{3}$, Deniz Çağdaş-Ayvaz ${ }^{1}$, Özden \\ Sanal ${ }^{1}$, İlhan Tezcan ${ }^{1}$ \\ ${ }^{1}$ Division of Pediatric Immunology and ${ }^{3}$ Department of Pediatrics, Hacettepe University Faculty of Medicine, Ankara; \\ ${ }^{2}$ Research Center for Translational Medicine, School of Medicine, Koc University, Istanbul. E-mail: baranerman@gmail.com \\ Received: 15th March 2017, Revised: 1st May 2017, Accepted: 2nd May 2017
}

SUMMARY: Erman B, Demirtaş D, Bildik HN, Çağdaş-Ayvaz D, Sanal Ö, Tezcan İ. Defective pneumococcal antibody response in patients with recurrent respiratory tract infections. Turk J Pediatr 2017; 59: 555-560.

Streptococcus pneumoniae is a common pathogen responsible for pulmonary infections and the leading cause of mortality and morbidity in patients with particularly B cell immunodeficiencies. Antibody production is the principal protective immune response against $S$. pneumoniae and measurement of the production of antipolysaccharide antibodies is important in the evaluation of B cell deficiencies. We quantified serotype-specific immunoglobulin G antibodies against seven common pneumococcal serotypes before and three weeks after unconjugated vaccine in 416 patients with recurrent respiratory tract infections; fifty-five (13\%) of whom showed impaired antibody response. We could evaluate 41 of these 55 patients for their particular clinical features. Specific antibody deficiency, was diagnosed in 10 of these patients, common variable immunodeficiency in 18, ataxia telangiectasia in 10 and other antibody deficiencies in 7 (transient hypogammaglobulinemia in 4, IgG subclass deficiency in 1, partial and selective IgA deficiency in 1) patients. Evaluation of the antibody response to polysaccharide antigens should be considered early on in patients with recurrent respiratory infections and required particularly for the diagnosis of specific antibody deficiency and the decision of the appropriate treatment approaches.

Key words: Streptococcus pneumoniae, respiratory tract infections, specific antibody response.

Antibodies are the primary mediators of humoral immunity against all classes of microbial antigens. Antigen molecules are usually proteins and polysaccharides, less frequently also lipids and nucleic acids. Coats, capsules, cell walls and toxins are antigenic parts of various organisms such as viruses and bacteria. Different types of antigens trigger different effector mechanisms of the immune system. B lymphocytes can recognize polysaccharide antigens by membrane bound immunoglobulin $\mathrm{M}$ and $\mathrm{D}$ receptors and $\mathrm{B}$ cell activation may occur in a $\mathrm{T}$ cell independent manner. Antibody responses to protein antigens require helper $\mathrm{T}$ lymphocytes and this is called $\mathrm{T}$ cell dependent process.

Antibody deficiency presents in both B and $\mathrm{T}$ cell disorders and is characterized by recurrent bacterial infections. ${ }^{1}$ Many primary immunodeficiencies predispose the individuals to infections with polysaccharide containing organisms. Streptococcus pneumoniae which is an encapsulated bacteria bearing surface polysaccharide antigens is the most common pathogen responsible for pulmonary infections. It is a clinically important pathogen that causes invasive pneumococcal diseases, such as sepsis, meningitis, and pneumonia. ${ }^{2-4}$ Measurement of specific antibody response is important for the evaluation and/or diagnosis of humoral immune disorders such as common variable immunodeficiency (CVID), selective IgA deficiency, IgG subclass deficiency and specific antibody deficiency (SAD). ${ }^{5}$ While impaired antibody production to various antigens are seen in many B cell immunodeficiencies, 
SAD is characterized by an impaired antibody production against polysaccharide antigens in the presence of normal immunoglobulins and normal response to protein antigens. Patients with SAD are usually prone to recurrent respiratory tract infections. ${ }^{6}$

In this study, we measured the polysaccharide antibody production in 416 patients with recurrent respiratory tract infections before and after unconjugated pneumococcal vaccine. Fifty-five patients were found to have impaired antibody response. Forty-one of these patients that we could reach were evaluated in terms of their clinical presentations.

\section{Material and Methods}

Four hundred and sixteen patients who were admitted with recurrent upper and/or lower respiratory tract infections enrolled according to 10 warning signs of primary immunodeficiencies by JMF (Jeffrey Modell Foundation) with or without hypogammaglobulinemia were immunized with polyvalent unconjugated pneumococcal vaccine between January 2008-December 2015. All patients were older than 2 years of age. Forty-one patients out of 55 with impaired antibody response could be included for clinical and immunological assessment in the study. In addition, among 416 we randomly selected 41 patients among 416 with intact vaccine response and without any known PID as a control group.

This study has been approved by the Local Ethics Committee of Hacettepe University (14.02.2017, report number: GO 17/86-14). Informed consent was obtained from patients and parents.

\section{Methods}

Measurement of capsular polysaccharide serotype-specific immunoglobulin G ( $\operatorname{IgG}$ ) antibodies against $S$. pneumoniae was performed by enzyme-linked immunosorbent assay (ELISA) in serum samples before and 4 weeks after vaccination with PNEUMO $23 \AA$ (unconjugated polysaccharide vaccine against S. pneumoniae) as described previously with minor modifications ${ }^{7}$. Antibody responses to 7 serotypes $(1,3,4,6 \mathrm{~B}, 14,19 \mathrm{~F}, 23 \mathrm{~F})$ were measured. All sera were obtained before starting IVIG therapy and analyzed in duplicates in the same ELISA run. In order to eliminate antibodies to cell wall polysaccharides, microtiter plates were coated with capsular polysaccharide antigens (from the American Type Culture Collection (ATCC), Rockville, MD, USA) and the samples were pre-incubated overnight with species-specific common cell wall polysaccharide from $S$. pneumoniae (CWPS; C-polysaccharide purified; Statens Serum Institute, Denmark). Antibody concentrations are indicated as the percentage of reference serum, the hyperimmune plasma pool (U.S. Pneumococcal Reference serum FDA7 CBER, Bethesda, MD, USA) in units per milliliter $(\mathrm{U} / \mathrm{ml})$, where the reference plasma pool represents $100 \mathrm{U} / \mathrm{ml}$ for each serotype. Since patients with high pre-immunization titers may not generate a drastic increase after immunization, the final concentration of antibodies after immunization (regardless of increase from preimmunization concentration) was taken into account ${ }^{8}$. Under an antibody concentration of less than $20 \mathrm{U} / \mathrm{ml}$ against a serotype tested was considered as a negative response and if negative response was obtained against at least $50 \%$ of the evaluated serotypes, it was considered as impaired response. This criterion was selected according to results obtained in 40 healthy Turkish children (age range: 5 to 15 years; median: 10 years and mean, 9.7 years) (Sanal O, unpublished data).

\section{Statistical analysis}

Comparisons between the patient groups with impaired antibody response and the controls were analyzed with Chi-square tests. Pearson, continuity correction or Fisher's exact test were used according to number of the subjects or expected values. SPSS Statistic 22 (IBM, USA) was used to analyze the data.

\section{Results}

Antibody production against polysaccharide antigens was defective in 55 patients out of 416 (13\%) with recurrent respiratory tract infections; 41 of these patients were evaluated.

The underlying immunodeficiencies were diagnosed according to criteria described by European Society for Immunodeficiencies. ${ }^{9,10}$ Ten patients were diagnosed with SAD, 18 with CVID, six with AT and seven with other antibody deficiencies (four patients with 
Table I. Age, Gender and Parental Consanguinity Rates of the Patient and Control Groups.

\begin{tabular}{|c|c|c|c|c|c|c|}
\hline \multirow[t]{2}{*}{ Diseases } & \multicolumn{4}{|c|}{ Age (years) } & \multirow{2}{*}{$\begin{array}{c}\text { Gender, } \\
\text { male/ female }\end{array}$} & \multirow{2}{*}{$\begin{array}{c}\text { Consanguinity, } \\
\%\end{array}$} \\
\hline & Mean & Median & SD & Range & & \\
\hline $\operatorname{SAD}(n=10)$ & 9.6 & 8 & \pm 3.7 & $6-18$ & $6 / 4$ & 40 \\
\hline CVID $(n=18)$ & 20.8 & 19.5 & \pm 11.4 & $5-48$ & $12 / 6$ & 16.7 \\
\hline $\operatorname{AT}(n=6)$ & 11.1 & 11.5 & \pm 5.0 & $5-18$ & $3 / 3$ & 66.7 \\
\hline OTHER $(\mathrm{n}=7)$ & 14.1 & 15 & \pm 5.8 & $7-22$ & $3 / 4$ & 42.9 \\
\hline $\begin{array}{l}\text { CONTROL } \\
(\mathrm{n}=41)\end{array}$ & 13.2 & 11 & \pm 7.8 & $4-32$ & $21 / 20$ & 14.6 \\
\hline
\end{tabular}

SAD: specific antibody deficiency, CVID: common variable immunodeficiency, AT: ataxia-telangiectasia

transient hypogammaglobulinemia, one IgG subclass deficiency, one partial IgA deficiency and one selective IgA deficiency). Age, gender and the rate of parental consanguinity were given in Table I. The patients were evaluated for the presence of recurrent lower and upper respiratory tract infections, bronchiectasis, autoimmunity, the number of hospitalizations due to the infections and the age of onset of clinical symptoms.

We compared the clinical and immunological features of patients with impaired antibody response against polysaccharide antigen with those of the control group. The patients with defective antibody response were divided into four groups as SAD, CVID, AT and the other hypogammaglobulinemies for further comparison (Table II).

The percentage of the patients with lower respiratory tract infections was much higher in SAD and CVID patients in comparison with the control group while excess upper respiratory tract infections were comparable in all groups $(p<0.001)$. Bronchiectasis demonstrated by chest X-ray or CT scan was present in half of the patients with SAD and CVID while it was present in $19 \%$ in the control group $(p=0,009)$.
The frequency of multiple hospitalization was similar in all patient groups (range $60-71 \%$ ) but it was much lower in the controls $(\mathrm{p}<0.001)$. Autoimmunity was most common in patients with CVID. However, the differences between patient groups and controls was not significant $(p=0.35)$. Defective antibody production against protein antigens was seen in patients with CVID and other hypogammaglobulinemies $(p=0.002)$. The mean age of onset of clinical symptoms was 56.7 months in the patients with impaired antibody response and 90.1 months in the control group.

\section{Discussion}

Measurement of polysaccharide antibody response is important in the evaluation of $B$ cell immunity. It should be evaluated in patients older than 2 years with recurrent bacterial infections. ${ }^{10}$ Impaired polysaccharide antibody production may be seen in antibody deficiencies such as CVID, IgG subclass deficiency and selective IgA deficiency. These patients may also have defective response to protein antigens. ${ }^{11-13}$ Patients with SAD have an impaired antibody production against polysaccharide antigens whereas they have

Table II. Clinical Features of the Patient and Control Groups.

\begin{tabular}{llllll}
\hline Clinical feature & SAD & CVID & AT & Other & Control \\
\hline URI & 70 & 89 & 67 & 86 & 63 \\
Pulmonary infection & 90 & 89 & 67 & 57 & 41 \\
Multiple hospitalization & 60 & 61 & 67 & 71 & 19 \\
Bronchiectasis & 50 & 50 & 0 & 0 & 19 \\
Autoimmunity & 10 & 39 & 0 & 14 & 7 \\
Response to protein antigens & 100 & 50 & 100 & 86 & 100
\end{tabular}

Data is presented as percentages. SAD: specific antibody deficiency, CVID: common variable immunodeficiency, AT: ataxia-telangiectasia, URI: upper respiratory tract infection. 
normal immunoglobulin levels and normal response to protein antigens. ${ }^{14}$

In this study, polysaccharide antibody response was found to be impaired in $13 \%$ of the patients with recurrent respiratory tract infections. All of the patients except one failed to respond to all 6 serotypes. One patient responded to only one serotype.

The percentage of the patients with recurrent lower respiratory tract infections was significantly higher in CVID and SAD (more than 2 fold) in comparison with the control group. The increased frequency of these infections was observed in AT and the other group, as well. Patients with CVID and selective IgA deficiency are more prone to recurrent lower respiratory tract infections ${ }^{15}$. Multiple hospitalization with respiratory tract infections in the patient groups were significantly higher than the control group which may suggest that the control group patients experience less severe infections.

Recurrent respiratory tract infections may result in bronchiectasis. ${ }^{16}$ It is one of the major long-term clinical complications in patients with SAD and CVID. ${ }^{17}$ Bronchiectasis was present in $50 \%$ of the patients with SAD and CVID but none of our patients with AT developed bronchiectasis. Recurrent lower respiratory tract infections are common in AT and can be associated with the development of bronchiectasis. ${ }^{18}$ A recent report from Iran showed that $54 \%$ of the patients had a history of pneumonia while $64 \%$ had a history of recurrent upper respiratory tract infections. ${ }^{19}$ Another study from the USA revealed that $38 \%$ of the patients over 20 years of age had a history of recurrent lower respiratory tract infections. ${ }^{20}$ Absence of AT patients with bronchiectasis in our study may result from the low number of patients and relatively young age. Lung disease in patients with AT often progresses with age and neurological decline and in addition to abnormal immune response, recurrent aspiration and impaired clearance of respiratory tract secretion contribute to lung disease in these patients ${ }^{21}$.

The parental consanguinity rate was higher in all patient groups except CVID in comparison the control group. This rate was $40 \%$ in patients with SAD which may be considered as notable. There is no data in the literature regarding consanguinity rate for these patients. The pathogenesis of SAD is still unclear. The underlying genetic defect in at least some of the patients may be inherited as autosomal recessive. Moreover, Turkey has a high rate of consanguineous marriages; some $20-25 \%$ of marriages in urban areas and more than $35 \%$ in rural areas are consanguineous. ${ }^{22}$ This may also explain the high ratio among our patients with SAD.

As expected percentage of patients with defective antibody production against protein antigens was $50 \%$ in the CVID group. It was the highest rate among all other patient and the control groups.

Autoimmunity was present most commonly in the CVID group. More than $25 \%$ of CVID patients have been reported to have autoimmune complications. ${ }^{23}$ Autoantibodies and auto-reactive B cells can be detected in these patients, even if serum immunoglobulins are very low and specific response to various antigens is impaired. ${ }^{24}$ Cytopenia is the most common autoimmune manifestation in these patients with CVID $^{25}$ and autoimmune thrombocytopenia (ITP) was found in 7 out of 18 patients in our study. One patient with IgG subclass deficiency had celiac disease.

Our results showed, clinical symptoms begin earlier in the patients with impaired antibody response in comparison with the control group who had recurrent respiratory tract infections but normal polysaccharide antibody production. Defective polysaccharide antibody production may be responsible at least partially for the earlier onset in patient groups than controls.

Jeurissen et al. ${ }^{26}$ found the prevalence of the impaired antibody production in the patients with recurrent respiratory tract infections to be $11 \%$ among 82 subjects. This ratio was $15 \%$ $(15 / 99)$ in the study of Ruuskanen et $\mathrm{al}^{27}$. Polysaccharide antibody response was found to be impaired in $13 \%(55 / 416)$ of our patients.

The prevalence of SAD among the patients with recurrent respiratory infections has been reported as $11 \%(10 / 91)$ and $12.4 \%(11 / 89)$ in the studies published by Rauskanen et al. ${ }^{27}$ and Boyle et al ${ }^{28}$, respectively. We found the prevalence of SAD was $2.4 \%(10 / 416)$. This is relatively lower than these previous results. Although our patients with SAD were younger 
than the CVID patients, the percentage of pulmonary infections and bronchiectasis were similar in patients with SAD and CVID. The difference between our and previous studies may be partly due to the fact that in the above mentioned studies the patients with low Ig levels were excluded.

In conclusion, measurement of the production of antipolysaccharide antibodies is required for diagnosis of SAD. Prophylactic antibiotic or IgG replacement therapy may protect these patients from long term complications. Thus, evaluation of the response to polysaccharide antigens should be considered early on in patients with recurrent respiratory infections.

\section{Acknowledgments}

The authors would like to thank the patients and their families for participating in the study. No financial assistance was received to conduct the study.

\section{REFERENCES}

1. Maarschalk-Ellerbroek LJ, Hoepelman IM, Ellerbroek PM. Immunoglobulin treatment in primary antibody deficiency. Int J Antimicrob Agents 2011; 37: 396-404.

2. Chaguza C, Cornick JE, Everett DB. Mechanisms and impact of genetic recombination in the evolution of Streptococcus pneumoniae. Comput Struct Biotechnol J 2015; 13: 241-247.

3. Jauneikaite E, Tocheva AS, Jefferies JM, et al. Current methods for capsular typing of Streptococcus pneumoniae. J Microbiol Methods 2015; 113: 41-49.

4. Gaschignard J, Levy C, Chrabieh M, et al. Invasive pneumococcal disease in children can reveal a primary immunodeficiency. Clin Infect Dis 2014; 59: 244-251.

5. Paris K, Sorensen RU. Assessment and clinical interpretation of polysaccharide antibody responses. Ann Allergy Asthma Immunol 2007; 99: 462-464.

6. Ochs HD, Stiehm ER, Winkelstein JA. Antibody deficiencies. Immunologic Disorders in Infants \& Children (5th ed). Philadelphia, 2004.

7. Sanal O, Ersoy F, Tezcan I, et al. Antibody response to a seven-valent pneumococcal conjugated vaccine in patients with ataxia-telangiectasia. J Clin Immunol 2004; 24: 411-417.

8. Santos-Valente E, Reisli I, Artaç H, et al. A novel mutation in the complement component 3 gene in a patient with selective IgA deficiency. J Clin Immunol 2013; 33: 127-133.

9. Conley ME, Notarengelo LD, Etzioni A. Diagnostic criteria for primary immunodeficiencies: Representing PAGID (Pan-American Group for Immunodeficiency) and ESID (European Society for Immunodeficiencies) Clin Immunol 1999; 93: 190-197.

10. Ballow M, Notarangelo L, Grimbacher B, et al Immunodeficiencies. Clin Exp Immunol 2009; 158: 14-22.

11. Tabatabaie P, Aghamohammadi A, Mamishi S, et al Evaluation of humoral immune function in patients with bronchiectasis. Iran J Allergy Asthma Immunol 2008; 7: 69-77.

12. Bossuyt X, Moens L, Van Hoeyveld E, et al. Coexistence of (partial) immune defects and risk of recurrent respiratory infections. Clin Chem 2007; 53: 124-130.

13. Salzer U, Warnatz K, Peter HH. Common variable immunodeficiency: an update. Arthritis Res Ther 2012; 14: 223

14. Cheng YK, Decker PA, O’Byrne MM, Weiler CR. Clinical and laboratory characteristics of 75 patients with specific polysaccharide antibody deficiency syndrome. Ann Allergy Asthma Immunol 2006; 97: 306-311.

15. Hurst JR, Workman S, Garcha DS, et al. Activity, severity and impact of respiratory disease in primary antibody deficiency syndromes. J Clin Immunol 2014; 34: 68-75.

16. Sidhu MK, Mandal P, Hill AT. Bronchiectasis: An update on current pharmacotherapy and future perspectives. Expert Opin Pharmacother 2014; 15: 505-525.

17. Verma N, Grimbacher B, Hurst JR. Lung disease in primary antibody deficiency. Lancet Respir Med 2015; 3: $651-660$

18. Boder E, Sedgwick JB. Ataxia-telangiectasia: A review of 101 cases. In: Walsh G, (ed). Little Club Clinics in Developmental Medicine. London: Heineman Medical Books, 1963; 110-118.

19. Moin M, Aghamohammadi A, Kouhi A, et al. Ataxiatelangiectasia in Iran: Clinical and laboratory features of 104 patients. Pediatr Neurol 2007; 37: 21-28.

20. Nowak-Wegrzyn A, Crawford TO, Winkelstein JA et al. Immunodeficiency and infections in ataxiatelangiectasia. J Pediatr 2004; 144: 505-511.

21. McGrath-Morrow SA, Gower WA, Rothblum-Oviatt $\mathrm{C}$, et al. Evaluation and management of pulmonary disease in ataxia-telangiectasia. Pediatr Pulmonol 2010; 45: 847-859.

22. Sanal O, Tezcan I. Thirty years of primary immunodeficiencies in Turkey. Ann N Y Acad Sci 2011; 1238: 15-23.

23. Baldovino S, Montin D, Martino S, et al. Common variable immunodeficiency: Crossroads between infections, inflammation and autoimmunity. Autoimmun Rev 2013; 12: 796-801.

24. Xiao X, Miao Q, Chang C, et al. Common variable immunodeficiency and autoimmunity--an inconvenient truth. Autoimmun Rev 2014; 13: 858-864

25. Agarwal S, Cunningham-Rundles C. Autoimmunity in common variable immunodeficiency. Curr Allergy Asthma Rep 2009; 9: 347-352. 
26. Jeurissen A, Moens L, Raes M, et al. Laboratory diagnosis of specific antibody deficiency to pneumococcal capsular polysaccharide antigens. Clin Chem 2007; 53: 505-510.

27. Ruuskanen O, Nurkka A, Helminen M, et al. Specific antibody deficiency in children with recurrent respiratory infections: a controlled study with followup. Clin Exp Immunol 2013; 172: 238-244
28. Boyle RJ, Le C, Balloch A, Tang Ml. The clinical syndrome of specific antibody deficiency in children. Clin Exp Immunol 2006; 146: 486-492. 